\title{
Nonlinear Forced Response of Electromechanical Integrated Toroidal Drive to Coupled Excitation
}

\author{
Lizhong Xu and Fen Wang \\ Mechanical Engineering Institute, Yanshan University, Qinhuangdao 066004, China \\ Correspondence should be addressed to Lizhong Xu, xlz@ysu.edu.cn
}

Received 27 May 2012; Accepted 24 July 2012

Academic Editors: K. Prokeš and B. Yasilata

Copyright () 2012 L. Xu and F. Wang. This is an open access article distributed under the Creative Commons Attribution License, which permits unrestricted use, distribution, and reproduction in any medium, provided the original work is properly cited.

The electric excitation and the parameter excitation from mesh stiffness fluctuation are analyzed. The forced response equations of the drive system to the coupled excitations are presented. For the exciting frequencies far from and near natural frequencies, the forced responses of the drive system to the coupled excitations are investigated. Results show that the nonlinear forced responses of the drive system to the coupled excitations change periodically and unsteadily; the time period of the nonlinear forced responses depends on the frequencies of the electric excitation, the mesh parameter excitation, and the nonlinear natural frequencies of the drive system; in order to improve the dynamics performance of the drive system, the frequencies of the electric excitations should not be taken as integral multiple of the mesh parameter exciting frequency.

\section{Introduction}

Toroidal drive can transmit large torque in a very small size and is suitable for technical fields such as aviation and space flight [1-3]. As electrical and control techniques are utilized in mechanical engineering field widely, generalized composite drives become advancing edge of the mechanical science. So far, types of the generalized composite drives with integrated structure are still very limited.

The electromagnetic harmonic drive [4] and piezoelectric harmonic drive [5] are active drives in which the meshing forces between flexible gear and rigid one are controlled by electromagnetic force or piezoelectric one, and drive and power are integrated. Based on researching toroidal drive [6], the authors presented a kind of active generalized composite drive: electromechanical integrated toroidal drive. In the drive, the toroidal drive, power, and control are integrated [7].

The drive consists of four basic elements (Figure 1) (a) the central worm, (b) radially positioned planets, (c) a toroidal shaped stator, and (d) a rotor, which forms the central output shaft upon which the planets are mounted. The central worm is fixed and coils are mounted in helical grooves of its surface. The planets have permanent magnets instead of teeth. The $N$ and $S$ polar permanent magnets are mounted alternately on a planet. And the stator has helical permanent magnets instead of helical teeth. In the same manner as planet, the $N$ and $S$ polar helical permanent magnets are mounted alternately on the stator.

If a specific parameter relation is realized, $N$ pole of one element will correspond to $S$ pole of the other one all along. The attractive forces between $N$ and $S$ pole of the different elements are driving forces and the meshes without contact are realized. When the alternating voltage source is connected to the coils of the worm, a toroidal circular field is formed. It drives several planets to rotate about their own axial. And by means of magnetic forces between teeth of the planet and stator, the rotor is driven to rotate about its own axial. Thus, a power of low speed and large torque is output.

Compared with toroidal drive, the new drive is easy to produce, without wear, and does not need lubrication. It can be substituted for a servo system to simplify the structure of the existing electromechanical systems. Beside the abovementioned fields that require compactness, the drive can be used in fields such as robots, which require accurate control.

The electromechanical integrated toroidal drive consists of a mechanical system, an electrical system and a coupled part. The mechanical vibration may occur in the mechanical 


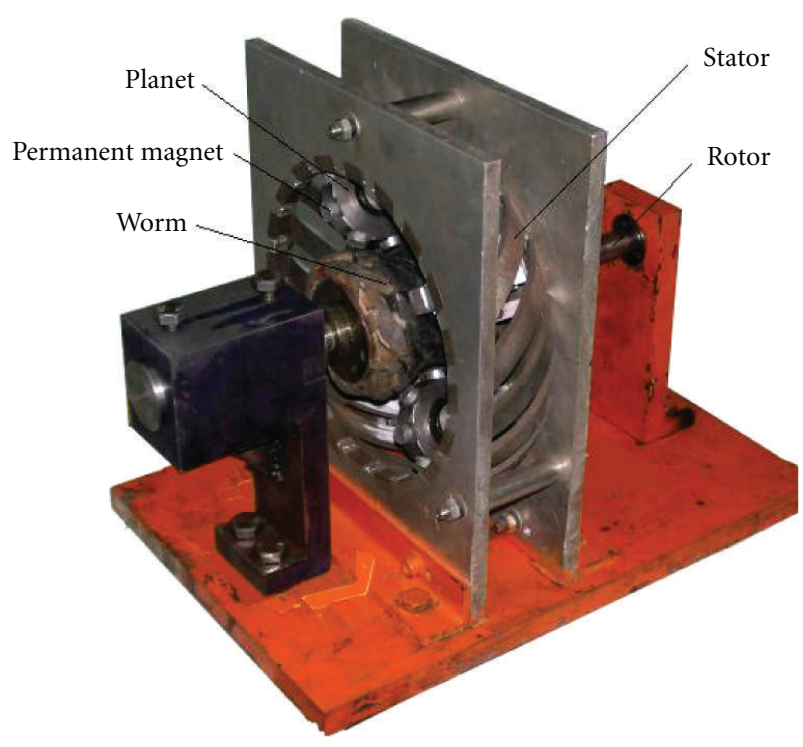

FIGURE 1: The electromechanical integrated toroidal drive.

system, and the electrical current oscillation may occur in the electrical system. With the coupled part, the mechanical vibration and the electrical current oscillation will influence on each other. Hence, the drive system is an electromechanical coupled dynamics system. The electromechanical coupled dynamics was first proposed for the motor [8]. Then, the electromechanical coupled dynamics of the electromechanical system consisting of the motor and mechanical system driven by the motor was developed. An electromechanical coupled dynamics model of the electromechanical system consisting of the several motors and mechanical system driven by these motors was proposed. Using the model, the natural frequency of the electromechanical system is analyzed [9]. The authors investigated nonlinear forced response of electromechanical integrated toroidal drive to voltage excitations [10]. However, not only the electric excitation occurs, but also the parameter excitations from mesh stiffness fluctuations occur. The nonlinear forced responses of the drive system to the coupled excitations consisting of the electric excitation and the parameter excitations have not been investigated yet. These nonlinear forced responses have important influence on the operating performance of the drive system. To design, evaluate, and control dynamics behavior of the drive system effectively, the nonlinear forced responses of the drive system to the coupled excitations should be developed.

In this paper, the electric excitation and the parameter excitation from mesh stiffness fluctuation are analyzed. The forced response equations of the drive system to the coupled excitations are presented. For the exciting frequencies far from and near natural frequencies, the forced responses of the drive system to the coupled excitations are investigated. The work can be used to predict the noise and dynamic load and are useful in maximizing the power density of the drive and reducing noise radiation.

\section{Electric Excitation of the Drive System}

The magnetic energy storage of the electric system for the drive system is

$$
W=\frac{1}{2} \sum_{i=1}^{n} \lambda_{i s} I_{i s}
$$

where $\lambda_{i s}$ is magnetic linkage of the worm coils, $\lambda_{i s}=$ $\sum_{j=1}^{n} L_{i j} I_{i s}, L_{i j}$ is inductances of the $i$ th phase worm coils, $I_{i s}$ is current of the $i$ th phase worm coils, and $n$ is phase number of the worm coils.

From (1), the electromagnetic torque on the planet is given as

$$
T_{p}=-\frac{\partial W}{\partial \theta}=-\frac{1}{2} \sum_{j=1}^{n} \sum_{i=1}^{n} \frac{d L_{i j}}{d \theta} I_{i s} I_{j s}
$$

where $\theta$ is the relative rotating angle between planet and worm.

The torque $T_{p}$ consists of the static torque $T_{p 0}$ and the dynamic torque $\delta T_{p}$. The current $I_{i s}$ consists of the static component $I_{i}$ and the dynamic one $\delta i_{i}$. The angle $\theta$ consists of the static angle $\theta_{0}$ and the dynamic angle $\delta \theta$. The torque $T_{p}$ is written in series form as

$$
\begin{aligned}
& T_{p}=T_{p 0}+\delta T_{p} \\
& =\frac{1}{2} \sum_{j=1}^{n} \sum_{i=1}^{n}\left[\left(\frac{\delta L_{i j}}{\delta \theta}\right)_{\theta=\theta_{0}} I_{i} I_{j}+\left(\frac{\delta^{2} L_{i j}}{\delta \theta^{2}}\right)_{\theta=\theta_{0}} I_{i} I_{j} \delta \theta\right. \\
& +\left(\frac{\delta^{3} L_{i j}}{\delta \theta^{3}}\right)_{\theta=\theta_{0}} I_{i} I_{j}(\delta \theta)^{2}+\left(\frac{\delta L_{i j}}{\delta \theta}\right)_{\theta=\theta_{0}} I_{i} \delta i_{j} \\
& +\left(\frac{\delta L_{i j}}{\delta \theta}\right)_{\theta=\theta_{0}} I_{j} \delta i_{i}+\left(\frac{\delta L_{i j}}{\delta \theta}\right)_{\theta=\theta_{0}} I_{i}\left(\delta i_{j}\right)^{2} \\
& \left.+\left(\frac{\delta L_{i j}}{\delta \theta}\right)_{\theta=\theta_{0}} I_{j}\left(\delta i_{i}\right)^{2}+\cdots\right] .
\end{aligned}
$$

If $\delta i_{j}=\delta i_{i}=0$, from (3), one can give

$$
\begin{gathered}
T_{p 0}=\frac{1}{2} \sum_{j=1}^{n} \sum_{i=1}^{n}\left(\frac{\delta L_{i j}}{\delta \theta}\right)_{\theta=\theta_{0}} I_{i} I_{j}, \\
\delta T_{p}=\frac{1}{2} \sum_{j=1}^{n} \sum_{i=1}^{n}\left[\left(\frac{\delta^{2} L_{i j}}{\delta \theta^{2}}\right)_{\theta=\theta_{0}} I_{i} I_{j} \delta \theta\right. \\
\left.+\left(\frac{\delta^{3} L_{i j}}{\delta \theta^{3}}\right)_{\theta=\theta_{0}} I_{i} I_{j}(\delta \theta)^{2}+\cdots\right] .
\end{gathered}
$$

Let $\Delta F_{w p i}$ denote the dynamic magnetic meshing force between a planet tooth and worm, $\Delta F_{w p i}=\delta T_{p} / R$. From (5), neglecting the high-order terms, one knows.

$$
\Delta F_{w p i}=-\frac{R \delta \theta}{2 R^{2}} \sum_{i=1}^{n} \sum_{j=1}^{n}\left[\left(\frac{\delta^{2} L_{i j}}{\delta \theta^{2}}\right)_{\theta=\theta_{0}} I_{i} I_{j}+\left(\frac{\delta^{3} L_{i j}}{\delta \theta^{3}}\right)_{\theta=\theta_{0}} I_{i} I_{j} \delta \theta\right] .
$$


Here, $\zeta_{w p i}=R \delta \theta$; it is the dynamic relative displacement between planet and worm. Thus, (6) can be changed into the following form:

$$
\Delta F_{w p i}=\left(k_{w p i}+\Delta k_{w p i}\right) \zeta_{w p i} .
$$

Here, $k_{w p i}=\left(1 / 2 R^{2}\right) \sum_{i=1}^{n} \sum_{i=1}^{n}\left(\delta^{2} L_{i j} / \delta \theta^{2}\right)_{\theta=\theta_{0}} I_{i s} I_{j s}$, it is considered as linear electromagnetic mesh stiffness between a tooth of the planet and worm; $\Delta k_{w p i}=$ $-\left(1 / 2 R^{2}\right) \sum_{i=1}^{n} \sum_{j=1}^{n}\left(\delta^{3} L_{i j} / \delta \theta^{3}\right)_{\theta=\theta_{0}} I_{i} I_{j} \delta \theta$, it is nonlinear component of the electromagnetic mesh stiffness.

Equation (3) shows that current fluctuation can produce electromagnetic torque fluctuation. The torque fluctuation from current fluctuation can be considered as equivalent exciting torque $\Delta T_{e}$. Let the current periodically change as below:

$$
\delta i_{i}=\delta i_{j}=\Delta I \cos \left(\omega_{e} t\right),
$$

where $\Delta I$ is the magnitude of the fluctuation current, $\omega_{e}$ is the frequency of the fluctuation current, and $t$ is time.

Substituting (8) into (3), neglecting high-order terms, yields

$$
\Delta T_{e}=\sum_{j=1}^{n} \sum_{i=1}^{n}\left(\frac{\delta L_{i j}}{\delta \theta}\right)_{\theta=\theta_{0}} I_{i} \Delta I \cos \omega_{e} t
$$

Let $\Delta F_{e}$ denote the equivalent exciting force between a planet tooth and worm; thus

$$
\Delta F_{e}=\frac{1}{R} \sum_{j=1}^{n} \sum_{i=1}^{n}\left(\frac{\delta L_{i j}}{\delta \theta}\right)_{\theta=\theta_{0}} I_{i} \Delta I \cos \omega_{e} t .
$$

\section{Parametric Excitation from Stiffness Fluctuation}

In operation of the drive system, the number of meshing tooth pairs between the worm and the planets is variable. It can cause fluctuation of the mesh stiffness between them. It is dependent on the number $z_{1}$ of planet teeth and the conditional face angles of the worm $\phi_{v}$. At $\phi_{v}=100^{\circ}$ and $z_{1}=8$, the changes of mesh stiffness along with the mesh tooth pair number are shown in Figure 2. In Figure 2, a typical stiffness variation through a mesh cycle of the drive system is given. The mesh stiffness at more contact regions is higher than that at less contact regions. If the drive rotates at appreciable speed, this time-varying stiffness will be a major excitation source of the drive system.

The mesh stiffness between a planet and worm is considered to consist of its mean value $\bar{k}$ and time-varying one $\Delta k(t)$. The average mesh stiffness between them through one periodic time can be given by

$$
\bar{k}_{w p i}=\frac{4}{\pi}\left\{\int_{0}^{\pi / 36} k_{3} d \theta+\int_{\pi / 36}^{2 \pi / 9} k_{2} d \theta+\int_{2 \pi / 9}^{\pi / 4} k_{3} d \theta\right\},
$$

where $k_{2}=2\left(k_{w p i}+\Delta k_{w p i}\right)$ and $k_{3}=3\left(k_{w p i}+\Delta k_{w p i}\right)$; they are two and three teeth mesh stiffness, respectively.

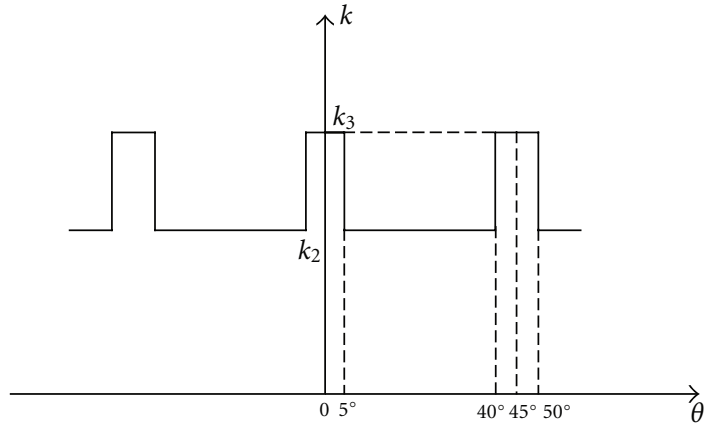

Figure 2: Changes of the mesh stiffness.

The periodical time-varying portion of the mesh stiffness can be defined in the Fourier series form as

$$
\Delta k(t)=\sum_{n=1}^{\infty} \Delta k_{n} \cos n \omega_{p} t
$$

where $\Delta k_{n}=(2 / l) \int_{0}^{l} k(t) \omega_{p} \cos n \omega_{p} t d t, l$ is the period of the stiffness fluctuation. In Figure $2, l=\pi / 4$.

For worm and planet, the periodical time-varying portion of the mesh stiffness is

$$
\begin{aligned}
\Delta k_{n w p i}=\frac{2}{l}\left\{\int_{0}^{\pi / 36}\left(k_{3}-\bar{k}_{w p i}\right) \omega_{p} \cos n \omega_{p} t d t\right. \\
+\int_{\pi / 36}^{2 \pi / 9}\left(k_{2}-\bar{k}_{w p i}\right) \omega_{p} \cos n \omega_{p} t d t \\
\left.\quad+\int_{2 \pi / 9}^{\pi / 4}\left(k_{3}-\bar{k}_{w p i}\right) \omega_{p} \cos n \omega_{p} t d t\right\} \\
=\frac{8}{\pi}\left[\left(k_{3}-\bar{k}_{w p i}\right)\left(\sin \frac{\pi}{36}+\sin \frac{\pi}{4}-\sin \frac{2 \pi}{9}\right)\right. \\
\left.\quad+\left(k_{2}-\bar{k}_{w p i}\right)\left(\sin \frac{2 \pi}{9}-\sin \frac{\pi}{36}\right)\right] .
\end{aligned}
$$

Substituting (13) into (12) yields

$$
\Delta k_{w p i}(t)=\sum_{n=1}^{\infty} \Delta k_{n w p i} \cos n \omega t .
$$

\section{Forced Response Equation to Coupled Excitation}

The dynamic model for the electromechanical integrated toroidal drive (see Figure 3) allows rotor and each planet to rotate about their own rotating axes and allows each planet to translate in $x_{i}$ and $z_{i}$ directions. The rotations are replaced by the corresponding translational mesh displacements as $u_{j}=r_{j} \theta_{j}, j=1, \ldots, m, r$ (here, $m$ is planet number, $\theta_{j}$ the rotation of planet or rotor, $r_{j}$ is the rolling circle radius for planet and the radius of the circle passing through planet centers for the rotor). A displacement vector $\mathbf{q}_{j}$ and a mass matrix $\mathbf{m}_{j}$ are defined for each planet $j$ as $\mathbf{q}_{j}=\left[\begin{array}{lll}u_{j} & x_{j} & z_{j}\end{array}\right]^{T}$ and $\mathbf{m}_{j}=\operatorname{Diag}\left[J_{j} / r_{j}^{2} \quad m_{j} m_{j}\right]$. Here, $J_{j}$ and $m_{j}$ are polar 
mass moment of inertia and mass for planet $j$, respectively. $M_{r}\left(M_{r}=J_{r} / r_{r}^{2}\right)$ is equivalent mass of rotor corresponding to its displacement $u_{r}$. Thus, the motion equations of the drive system are

$$
\begin{aligned}
& \frac{J_{i}}{r_{i}^{2}} \ddot{u}_{i}+\left(k_{w p i}+\Delta k_{w p i}\right) p_{w p i} \sin \gamma_{w p i} \\
& \quad+\Delta F_{e} \sin \gamma_{w p i}-k_{s p i} p_{s p i} \cos \gamma_{s p i}=0 \\
& m_{i} \ddot{x}_{i}+k_{c p x i} x_{i}=0, \\
& m_{i} \ddot{z}_{i}-\left(k_{w p i}+\Delta k_{w p i}\right) p_{w p i} \cos \gamma_{w p i} \\
& \quad-\Delta F_{e} \cos \gamma_{w p i}-k_{s p i} p_{s p i} \sin \gamma_{s p i}+k_{c p z i} p_{c p z i}=0, \\
& M_{r} \ddot{u}_{r}-\sum_{i=1}^{m} k_{c p z i} p_{c p z i}=-\frac{T_{r}}{r_{r}} \quad(i=1 \text { to } m),
\end{aligned}
$$

where $p_{w p i}, p_{s p i}$ and $p_{c p z i}$ are relative displacements between planet- $i$ and worm, stator, or rotor, respectively, $p_{w p i}=$ $u_{i} \sin \gamma_{w p i}-z_{i} \cos \gamma_{w p i}, p_{s p i}=-u_{i} \cos \gamma_{s p i}-z_{i} \sin \gamma_{s p i}$ and $p_{c p z i}=z_{i}-u_{r} \cdot \gamma_{w p i}$ and $\gamma_{s p i}$ are lead angles at contact points between planet- $i$ and worm or stator, respectively, $\tan \gamma_{w p i}=$ $1 /\left[i_{w p}(a / R-1)\right]$, and $\tan \gamma_{s p i}=1 /\left[i_{s p}(a / R+1)\right]$. Here, $a$ is center distance between worm and planet $R$ is reference circle radius of planet $i_{w p}$ and $i_{s p}$ are speed ratios between planet and worm or stator, respectively. $k_{w p i}$ and $k_{s p i}$ are mesh stiffness between planet- $i$ and worm or stator, respectively, $k_{c z i}$ and $k_{c x i}$ are planet support stiffness in $z_{i}$ and $x_{i}$ directions, respectively, and $T_{r}$ is torque transmitted by rotor.

Equation (15) can be written in matrix form as

$$
\mathbf{M X}+\mathbf{K X}=\mathbf{F}+\varepsilon \Delta \mathbf{F}+\varepsilon \Delta \mathbf{F}_{e},
$$

where $\mathbf{X}$ and $\mathbf{F}$ are displacement and static load vectors; respectively,

$$
\begin{aligned}
& \mathbf{X}=\left\{\begin{array}{lllll}
\mathbf{q}_{1} & \cdots & \cdots & \mathbf{q}_{m} & u_{r}
\end{array}\right\}^{T} \\
& \mathbf{F}=\left\{\begin{array}{lllll}
\mathbf{0} & \cdots & \cdots & \mathbf{0} & -T_{r} / r_{r}
\end{array}\right\}^{T}
\end{aligned}
$$

$\mathbf{M}$ and $\mathbf{K}$ are mass and stiffness matrix, respectively,

$$
\mathbf{M}=\operatorname{diag}\left[\begin{array}{lllll}
\mathbf{m}_{1} & \cdots & \cdots & \mathbf{m}_{m} & M_{r}
\end{array}\right],
$$

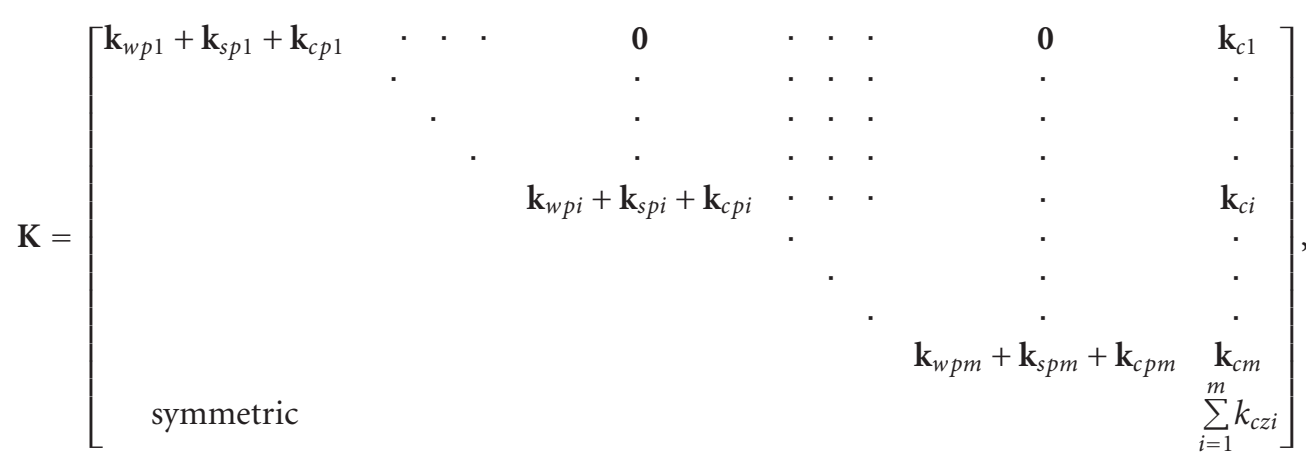

$$
\begin{gathered}
\mathbf{k}_{w p i}=k_{w p i}\left[\begin{array}{ccc}
\sin ^{2} \gamma_{w p i} & 0 & -\cos \gamma_{w p i} \sin \gamma_{w p i} \\
0 & 0 & 0 \\
-\cos \gamma_{w p i} \sin \gamma_{w p i} & 0 & \cos ^{2} \gamma_{w p i}
\end{array}\right], \\
\mathbf{k}_{s p i}=k_{s p i}\left[\begin{array}{ccc}
\cos ^{2} \gamma_{s p i} & 0 & \sin \gamma_{s p i} \cos \gamma_{s p i} \\
0 & 0 & 0 \\
\sin \gamma_{s p i} \cos \gamma_{s p i} & 0 & \sin ^{2} \gamma_{s p i}
\end{array}\right], \\
\mathbf{k}_{c p i}=\left[\begin{array}{ccc}
0 & 0 & 0 \\
0 & k_{c x i} & 0 \\
0 & 0 & k_{c z i}
\end{array}\right], \quad \mathbf{k}_{c i}=\left\{\begin{array}{lll}
0 & 0 & -k_{c z i}
\end{array}\right\}^{T} .
\end{gathered}
$$

$\Delta \mathbf{F}$ is nonlinear component of the force vector, $\Delta \mathbf{F}_{e}$ is equivalent force vector caused by current fluctuation:

\section{Consider}

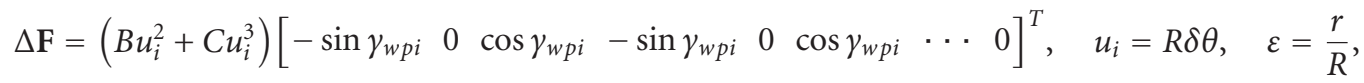


it is a disturbing parameter, $B=z_{1} \sum_{i=1}^{m} \sum_{j=1}^{m} A \tan \left(z_{1} \theta-\right.$

$\left.\phi_{v} / n p\right) I_{i} I_{j} / 2 R r, C=-z_{1}^{2} \sum_{i=1}^{m} \sum_{j=1}^{m} A I_{i} I_{j} / 2 R r$.

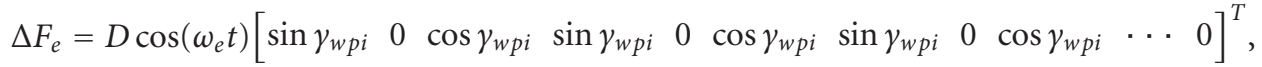

$$
\begin{aligned}
& D=\frac{1}{2 R} \sum_{i=1}^{n} \sum_{j=1}^{n}\left(\frac{\partial L_{i j}}{\partial \theta}\right)_{\theta=\theta_{0}} I_{i} \Delta I \text {. }
\end{aligned}
$$

The total displacement of the each component consists of the static displacement and the dynamic one. Hence, the displacement vector is

$$
\mathbf{X}=\overline{\mathbf{X}}+\Delta \mathbf{X},
$$

where $\overline{\mathbf{X}}$ and $\Delta \mathbf{X}$ are static and dynamic displacement vectors; respectively,

$$
\begin{aligned}
& \Delta \mathbf{X}=\left\{\begin{array}{lllll}
\Delta \mathbf{q}_{1} & \cdots & \cdots & \Delta \mathbf{q}_{m} & \Delta u_{r}
\end{array}\right\}^{T}, \\
& \Delta \mathbf{q}_{j}=\left[\begin{array}{lll}
\Delta u_{j} & \Delta x_{j} & \Delta z_{j}
\end{array}\right]^{T} .
\end{aligned}
$$

Considering the fluctuation of the mesh stiffness caused by the changes of the mesh tooth pair number, the stiffness matrix can be expressed as

$$
\mathbf{K}=\overline{\mathbf{K}}+\Delta \mathbf{K}(t),
$$

where $\overline{\mathbf{K}}$ is the mean stiffness matrix, and $\Delta \mathbf{K}(t)$ is timevarying stiffness matrix.

Substituting (20)-(22) into (16) yields

$$
\begin{gathered}
\overline{\mathbf{K}} \overline{\mathbf{X}}=\mathbf{F}, \\
\mathbf{M} \Delta \ddot{\mathbf{X}}+\overline{\mathbf{K}} \Delta \mathbf{X}=\varepsilon \Delta \mathbf{F}+\varepsilon \Delta \mathbf{F}_{e}+\varepsilon \Delta \mathbf{F}_{p},
\end{gathered}
$$

where $\Delta \mathbf{F}_{p}=-E \Delta \mathbf{K}(t) \overline{\mathbf{X}}$; it is considered as the equivalent exciting force vector caused by mesh stiffness excitation; $E=$ $R / r$.

Equation (24) is just the forced response equation of the drive system to coupled excitation.

\section{Solution of the Nonlinear Forced Response Equation}

5.1. Far from Natural Frequencies. First, we consider to resolve the nonlinear forced response equation of the drive system to electric excitation (let $\Delta \mathbf{F}_{p}=0$ ).

For simplicity purposes, (24) should be transmitted into equations independent on each other. Then, (24) is changed into the following form:

$$
\Delta \ddot{\mathbf{X}}_{N}+\overline{\mathbf{K}}_{N} \Delta \mathbf{X}_{N}=\varepsilon \Delta \mathbf{F}_{N}+\varepsilon \Delta \mathbf{F}_{e N},
$$

where $\overline{\mathbf{K}}_{N}$ is the diagonal mean stiffness matrix, respectively. $\Delta \mathbf{X}_{N}$ is transmitted dynamic displacement vector. $\Delta \mathbf{F}_{e N}$ and
$\Delta \mathbf{F}_{N}$ are transmitted exciting force vectors of the forces $\Delta \mathbf{F}_{e}$ and $\Delta \mathbf{F}$, respectively.

Matrix $\mathbf{K}_{N}$ and vector $\Delta \mathbf{X}_{N}$ are given by $\mathbf{K}_{N}=\mathbf{A}_{N}^{T} \mathbf{K A}_{N}$ and $\Delta \mathbf{X}_{N}=\mathbf{A}_{N}^{T} \Delta \mathbf{X}$. The transmitted nonlinear force vector $\Delta \mathbf{F}_{N}$ and transmitted equivalent exciting force vector $\Delta \mathbf{F}_{e N}$ can be given as below:

$$
\begin{gathered}
\Delta \mathbf{F}_{N}=\mathbf{A}_{N}^{T} \Delta \mathbf{F}=\left(B u_{i}^{2}+C u_{i}^{3}\right) \Delta \mathbf{P}_{N}, \\
\Delta \mathbf{F}_{e N}=\varepsilon D \cos \left(\omega_{e} t\right) \Delta \mathbf{P}_{e N},
\end{gathered}
$$

where $\mathbf{A}_{N}$ is the mode matrix of (24):

$$
\mathbf{A}_{N}=\left[\begin{array}{cccc}
A_{1}^{(1)} & A_{2}^{(1)} & \cdots & A_{m}^{(1)} \\
A_{1}^{(2)} & A_{2}^{(2)} & \cdots & A_{m}^{(2)} \\
\cdots & \cdots & \cdots & \cdots \\
A_{1}^{(m)} & A_{2}^{(m)} & \cdots & A_{m}^{(m)}
\end{array}\right] .
$$

At $m=3, \Delta \mathbf{P}_{N}=\left[\begin{array}{lllll}P_{N 1} & P_{N 2} & \cdots & P_{N i} & \cdots\end{array}\right]^{T}, \mathbf{P}_{N i}=$ $-3 A_{N 1}^{i} \sin \gamma_{w p i}+3 A_{N 3}^{i} \cos \gamma_{w p i}(i=1,4, \ldots, 3 m-2), P_{N i}=$ $0(i=2,3, \ldots, 3 m+1, i \neq 1,4, \ldots, 3 m-2) ; \Delta \mathbf{P}_{e N}=$ $\left[\begin{array}{llllllllll}P_{e N 1} & 0 & 0 & P_{e N 2} & 0 & 0 & P_{e N 3} & 0 & 0 & 0\end{array}\right]^{T}, P_{N e}=3 A_{N 1}^{i} \sin \gamma_{w p i}+$ $3 A_{N 3}^{i} \cos \gamma_{w p i}(i=1,4, \ldots, 3 m-2), P_{N e i}=0(i=2,3, \ldots, 3 m+$ $1, i \neq 1,4, \ldots, 3 m-2)$.

Let

$$
\begin{gathered}
\Delta \mathbf{X}_{N}=\mathbf{X}_{0}+\varepsilon \mathbf{X}_{1}+\varepsilon^{2} \mathbf{X}_{2}+\cdots, \\
\omega_{i}^{2}=\omega_{0 i}^{2}\left(1+\varepsilon \sigma_{1}^{i}+\varepsilon^{2} \sigma_{2}^{i}+\cdots\right) \quad(i=1,2,3),
\end{gathered}
$$

where $\omega_{i}$ is natural frequency of $i$ th order mode for the nonlinear drive system, and $\omega_{0 i}$ is natural frequency of $i$ th order rotational mode for the linear drive system.

Substituting (28) and (29) into (25), let sum of the coefficients with the same-order power of the parameter $\varepsilon$ equal zero, following equations can be given

$$
\begin{aligned}
\ddot{x}_{N 0}+\omega_{i}^{2} x_{N 0}= & 0, \\
\ddot{x}_{N 1}+\omega_{i}^{2} x_{N 1}= & -\sigma_{1} \ddot{x}_{N 0}+P_{N} B u_{0 i}^{2}+P_{N} C u_{0 i}^{3}+D \cos \left(\omega_{e} t\right) P_{N e} \\
\ddot{x}_{N 2}+\omega_{i}^{2} x_{N 2}= & -\sigma_{1} \ddot{x}_{N 1}-\sigma_{2} \ddot{x}_{N 0}+P_{N} B\left(2 u_{0 i} u_{1 i}+\sigma_{1} u_{0 i}^{2}\right) \\
& +P_{N} C\left(3 u_{0 i}^{2} u_{1 i}+\sigma_{1} u_{0 i}^{3}\right)+D \sigma_{1} \cos \left(\omega_{e} t\right) P_{N e}
\end{aligned}
$$




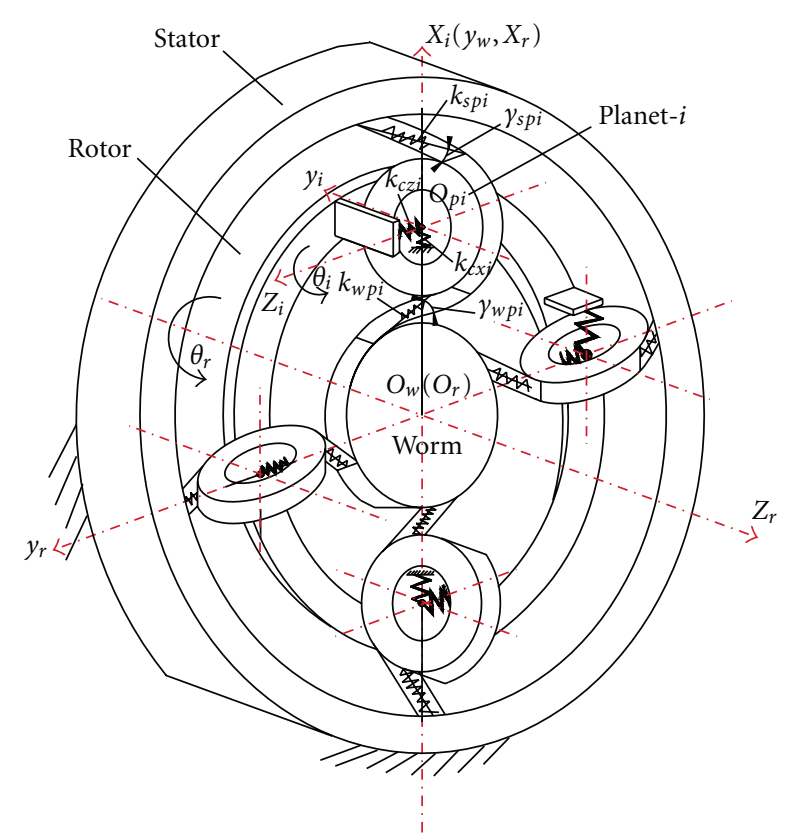

Figure 3: Dynamic model for a four-planet electromechanical integrated toroidal drive.

Here, initial conditions are $x_{N 0}^{i}(0)=A_{N 0}^{i}, \dot{x}_{N 0}^{i}(0)=0$.

The solution of zero-order equation under the above initial conditions is

$$
x_{0}^{i}=A_{0}^{i} \cos \omega_{i} t \quad(i=1,2,3) .
$$

Substituting (31) into the second equation of (30) yields

$$
\begin{aligned}
& \ddot{x}_{N 1}^{1}+\omega_{1}^{2} x_{N 1}^{1}=-\sigma_{1}^{1} \ddot{x}_{N 0}^{1}+P_{N 1} B u_{0 i}^{2}+P_{N 1} C u_{0 i}^{3}+D \cos \left(\omega_{e} t\right) P_{N e}, \\
& \ddot{x}_{N 1}^{2}+\omega_{2}^{2} x_{N 1}^{2}=-\sigma_{1}^{2} \ddot{x}_{N 0}^{2}+P_{N 2} B u_{0 i}^{2}+P_{N 2} C u_{0 i}^{3}+D \cos \left(\omega_{e} t\right) P_{N e}, \\
& \ddot{x}_{N 1}^{3}+\omega_{3}^{2} x_{N 1}^{3}=-\sigma_{1}^{3} \ddot{x}_{N 0}^{3}+P_{N 3} B u_{0 i}^{2}+P_{N 3} C u_{0 i}^{3}+D \cos \left(\omega_{e} t\right) P_{N e} .
\end{aligned}
$$

The rotational displacement $u_{i}$ is

$$
u_{i}=A_{N 1}^{1} x_{N i}^{1}+A_{N 1}^{2} x_{N i}^{2}+A_{N 1}^{3} x_{N i}^{3} .
$$

Substituting (33) into (30) yields

$$
\begin{aligned}
\ddot{x}_{N 1}^{1}+ & \omega_{1}^{2} x_{N 1}^{1} \\
= & -\sigma_{1}^{1} \ddot{x}_{N 0}^{1}+P_{N 1} B\left(A_{N 1}^{1} x_{N 0}^{1}+A_{N 1}^{2} x_{N 0}^{2}+A_{N 1}^{3} x_{N 0}^{3}\right)^{2} \\
& +P_{N 1} C\left(A_{N 1}^{1} x_{N 0}^{1}+A_{N 1}^{2} x_{N 0}^{2}+A_{N 1}^{3} x_{N 0}^{3}\right)^{3} \\
& +D \cos \left(\omega_{e} t\right) P_{N e 1}
\end{aligned}
$$

$$
\begin{aligned}
\ddot{x}_{N 1}^{2}+ & \omega_{2}^{2} x_{N 1}^{2} \\
= & -\sigma_{1}^{2} \ddot{x}_{N 0}^{2}+P_{N 2} B\left(A_{N 1}^{1} x_{N 0}^{1}+A_{N 1}^{2} x_{N 0}^{2}+A_{N 1}^{3} x_{N 0}^{3}\right)^{2} \\
& +P_{N 2} C\left(A_{N 1}^{1} x_{N 0}^{1}+A_{N 1}^{2} x_{N 0}^{2}+A_{N 1}^{3} x_{N 0}^{3}\right)^{3} \\
& +D \cos \left(\omega_{e} t\right) P_{N e 2}, \\
\ddot{x}_{N 1}^{3} & \omega_{3}^{2} x_{N 1}^{3} \\
= & -\sigma_{1}^{3} \ddot{x}_{N 0}^{3}+P_{N 3} B\left(A_{N 1}^{1} x_{N 0}^{1}+A_{N 1}^{2} x_{N 0}^{2}+A_{N 1}^{3} x_{N 0}^{3}\right)^{2} \\
& +P_{N 3} C\left(A_{N 1}^{1} x_{N 0}^{1}+A_{N 1}^{2} x_{N 0}^{2}+A_{N 1}^{3} x_{N 0}^{3}\right)^{3} \\
& +D \cos \left(\omega_{e} t\right) P_{N e 3} .
\end{aligned}
$$

In order to remove secular item, let

$$
\begin{aligned}
\sigma_{1}^{1}=- & \frac{P_{N 1} C}{\omega_{1}^{2}} \\
\times & {\left[\frac{3}{4}\left(A_{N 1}^{1} A_{N 0}^{1}\right)^{3}+\frac{3}{2}\left(A_{N 1}^{2} A_{N 0}^{2}\right)^{2} A_{N 1}^{1} A_{N 0}^{1}\right.} \\
& \left.+\frac{3}{2}\left(A_{N 1}^{3} A_{N 0}^{3}\right)^{2} A_{N 1}^{1} A_{N 0}^{1}\right], \\
\sigma_{1}^{2}=- & \frac{P_{N 2} C}{\omega_{2}^{2}} \\
\times & {\left[\frac{3}{4}\left(A_{N 1}^{2} A_{N 0}^{2}\right)^{3}+\frac{3}{2}\left(A_{N 1}^{1} A_{N 0}^{1}\right)^{2} A_{N 1}^{2} A_{N 0}^{2}\right.} \\
& \left.+\frac{3}{2}\left(A_{N 1}^{3} A_{N 0}^{3}\right)^{2} A_{N 1}^{2} A_{N 0}^{2}\right], \\
\sigma_{1}^{3}=- & \frac{P_{N 3} C}{\omega_{3}^{2}} \\
\times & {\left[\frac{3}{4}\left(A_{N 1}^{3} A_{N 0}^{3}\right)^{3}+\frac{3}{2}\left(A_{N 1}^{2} A_{N 0}^{2}\right)^{2} A_{N 1}^{3} A_{N 0}^{3}\right.} \\
& \left.+\frac{3}{2}\left(A_{N 1}^{1} A_{N 0}^{1}\right)^{2} A_{N 1}^{3} A_{N 0}^{3}\right] .
\end{aligned}
$$

Substituting (35) into (34), the solutions of the firstorder equations can be obtained. As the equations of the solutions are relatively complicated, it is not given here.

In a same manner, the solution of $n$th order equation can be obtained as well. Substituting these solutions into (28) and (29), the solutions of the regular nonlinear forced response equations and natural frequencies of the drive system can be given. Then, the real solutions of the nonlinear forced responses can be calculated as below:

$$
\Delta \mathbf{X}=\mathbf{A}_{N} \Delta \mathbf{X}_{N} .
$$

5.2. Near Natural Frequencies. When the exciting frequency is near natural frequency, the nonlinear forced response equation can be resolved as below. Considering damping of the drive system, (25) can be changed into

$$
\Delta \ddot{\mathbf{X}}_{N}+\mathbf{C}_{N} \Delta \dot{\mathbf{X}}_{N}+\mathbf{K}_{N} \Delta \mathbf{X}_{N}=\varepsilon \Delta \mathbf{F}_{N}+\varepsilon \Delta \mathbf{F}_{e N},
$$


where $\mathbf{C}_{N}$ is regular damping matrix, $\mathbf{C}_{N}=\mathbf{A}_{N}^{T} \mathbf{C} \mathbf{A}_{N}=$ $\operatorname{diag}\left[\begin{array}{llll}C_{N 1} & C_{N 2} & C_{N 3} & C_{N 4}\end{array}\right]$ ( $\mathbf{C}$ is damping matrix). $C_{N i}=$ $2 \zeta \omega_{i}, \zeta$ is relative damping coefficient.

When exciting frequency is near natural frequency, the exciting frequency can be written as

$$
\omega_{e i}^{2}=\omega_{0 i}^{2}\left(1+\varepsilon \sigma_{1}^{i}\right) \quad(i=1,2,3) .
$$

Substituting (38) and (28) into (37), let sum of the coefficients with the same-order power of the parameter $\varepsilon$ equal zero, the following equations can be given:

$$
\begin{aligned}
\ddot{\mathbf{X}}_{0}+\mathbf{K}_{N} \mathbf{X}_{0}= & 0, \\
\ddot{\mathbf{X}}_{1}+\mathbf{K}_{N} \mathbf{X}_{1}= & -\sigma_{1}^{i} \ddot{\mathbf{X}}_{0}-2 \zeta_{1} \dot{\mathbf{X}}_{0} \\
& +\Delta \mathbf{P}_{N}\left(B_{i} u_{i}^{2}+C_{i} u_{i}^{3}\right)+\Delta \mathbf{P}_{e N} D_{i} \cos \left(\omega_{e i} t+\theta\right) \\
& \cdots,
\end{aligned}
$$

where $C_{N i}=2 \zeta \omega_{i}$,

$$
\begin{gathered}
\zeta=\varepsilon \zeta_{1}, \\
\omega_{e i}^{2}=\omega_{i}^{2}\left(1+\varepsilon \sigma_{1}\right), \\
\Delta x_{N i}=x_{N 0 i}+\varepsilon x_{N 1 i}, \\
B=B_{i} \omega_{i}^{2}, \\
C=C_{i} \omega_{i}^{2}, \\
D=D_{i} \omega_{i}^{2} .
\end{gathered}
$$

The solution of zero-order equation under the above initial conditions is

$$
x_{N 0}^{i}=A_{N 0}^{i} \cos \omega_{e i} t \quad(i=1,2,3) .
$$

Substituting (41) into the second equation of (43) yields

$$
\begin{aligned}
\ddot{x}_{N 1}^{1}+\omega_{e 1}^{2} x_{N 1}^{1}= & -\sigma_{1} \ddot{x}_{N 0}^{1}-2 \zeta_{1} \dot{x}_{N 0}^{1}+P_{N 1} B_{1} u_{01}^{2}+P_{N 1} C_{1} u_{01}^{3} \\
& +D_{1} P_{N e 1} \cos \left(\omega_{e 1} t+\theta\right), \\
\ddot{x}_{N 1}^{2}+\omega_{e 2}^{2} x_{N 1}^{2}= & -\sigma_{1} \ddot{x}_{N 0}^{2}-2 \zeta_{1} \dot{x}_{N 0}^{2}+P_{N 2} B_{2} u_{01}^{2}+P_{N 2} C_{2} u_{01}^{3} \\
& +D_{2} P_{N e 2} \cos \left(\omega_{e 2} t+\theta\right), \\
\ddot{x}_{N 1}^{3}+\omega_{e 3}^{2} x_{N 1}^{3}= & -\sigma_{1} \ddot{x}_{N 0}^{3}-2 \zeta_{1} \dot{x}_{N 0}^{3}+P_{N 3} B_{3} u_{01}^{2}+P_{N 3} C_{3} u_{01}^{3} \\
& +D_{3} P_{N e 3} \cos \left(\omega_{e 3} t+\theta\right) .
\end{aligned}
$$

Substituting rotational displacement $u_{i}=A_{N 1}^{1} x_{N i}^{1}+$ $A_{N 1}^{2} x_{N i}^{2}+A_{N 1}^{3} x_{N i}^{3}$ into (42) yields

$$
\begin{aligned}
\ddot{x}_{N 1}^{1}+ & \omega_{e 1}^{2} x_{N 1}^{1} \\
= & -\sigma_{1} \ddot{x}_{N 0}^{1}-2 \zeta_{1} \dot{x}_{N 0}^{1}+P_{N 1} B_{1}\left(A_{N 1}^{1} x_{N 0}^{1}+A_{N 1}^{2} x_{N 0}^{2}+A_{N 1}^{3} x_{N 0}^{3}\right)^{2} \\
& +P_{N 1} C_{1}\left(A_{N 1}^{1} x_{N 0}^{1}+A_{N 1}^{2} x_{N 0}^{2}+A_{N 1}^{3} x_{N 0}^{3}\right)^{3} \\
& +D_{1} P_{N e 1}\left(\cos \omega_{e 1} t \cos \theta+\sin \omega_{e 1} t \sin \theta\right), \\
\ddot{x}_{N 1}^{2}+ & \omega_{e 2}^{2} x_{N 1}^{2} \\
= & -\sigma_{1} \ddot{x}_{N 0}^{2}-2 \zeta_{1} \dot{x}_{N 0}^{2}+P_{N 2} B_{2}\left(A_{N 1}^{1} x_{N 0}^{1}+A_{N 1}^{2} x_{N 0}^{2}+A_{N 1}^{3} x_{N 0}^{3}\right)^{2} \\
& +P_{N 2} C_{2}\left(A_{N 1}^{1} x_{N 0}^{1}+A_{N 1}^{2} x_{N 0}^{2}+A_{N 1}^{3} x_{N 0}^{3}\right)^{3} \\
& +D_{2} P_{N e 2}\left(\cos \omega_{e 2} t \cos \theta+\sin \omega_{e 2} t \sin \theta\right), \\
\ddot{x}_{N 1}^{3}+ & \omega_{e 3}^{2} x_{N 1}^{3} \\
= & -\sigma_{1} \ddot{x}_{N 0}^{3}-2 \zeta_{1} \dot{x}_{N 0}^{3}+P_{N 3} B_{3}\left(A_{N 1}^{1} x_{N 0}^{1}+A_{N 1}^{2} x_{N 0}^{2}+A_{N 1}^{3} x_{N 0}^{3}\right)^{2} \\
& +P_{N 3} C_{3}\left(A_{N 1}^{1} x_{N 0}^{1}+A_{N 1}^{2} x_{N 0}^{2}+A_{N 1}^{3} x_{N 0}^{3}\right)^{3} \\
& +D_{3} P_{N e 3}\left(\cos \omega_{e 3} t \cos \theta+\sin \omega_{e 3} t \sin \theta\right) .
\end{aligned}
$$

In order to remove secular item, let

$$
\begin{gathered}
\sigma_{1}^{1} A_{N 0}^{1}+P_{N 1} C_{1} A_{N 0}^{1} P_{1}^{\prime}+D_{1} P_{N e 1} \cos \theta=0, \\
2 \zeta_{1} A_{N 0}^{1}+D_{1} P_{N e 1} \sin \theta=0,
\end{gathered}
$$

where $P_{1}^{\prime}=\left(1 / A_{N 0}^{1}\right)\left[(3 / 4)\left(A_{N 1}^{1} A_{N 0}^{1}\right)^{3}+(3 / 2)\left(A_{N 1}^{2} A_{N 0}^{2}\right)^{2} A_{N 1}^{1} A_{N 0}^{1}+\right.$ $\left.(3 / 2)\left(A_{N 1}^{3} A_{N 0}^{3}\right)^{2} A_{N 1}^{1} A_{N 0}^{1}\right]$.

From (44), it is known that

$$
\left(\sigma_{1}^{1}+P_{N 1} C_{1} P_{1}^{\prime}\right)^{2}+\left(2 \zeta_{1}\right)^{2}=\left(\frac{D_{1} P_{N e 1}}{A_{N 0}^{1}}\right)^{2} .
$$

Thus

$$
\begin{aligned}
s_{i}^{2}=1 & -\varepsilon P_{N i} C_{i} P_{i}^{\prime}-2 \zeta^{2} \\
& \pm \sqrt{\left(\frac{D_{i}^{\prime}}{A_{N 0}^{i}}\right)^{2}-4 \zeta^{2}\left(1-\varepsilon P_{N i} C_{i} P_{i}^{\prime}-\zeta^{2}\right)},
\end{aligned}
$$

where $s_{i}=\omega_{e i} / \omega_{i}$ and $D_{i}^{\prime}=\varepsilon D_{i} P_{N e i}$.

From (46), the changes of the nonlinear vibrating magnitudes along with exciting frequencies can be given.

\section{Results and Discussions}

When exciting frequency is far from natural frequency, from the above equations, the nonlinear forced vibrations for the drive system are analyzed. The parameters of the numerical example are shown in Table 1. Figure 4 shows changes of the forced vibrations of the transmitted variables along with nonlinear parameter $\varepsilon$. From Figure 4, the following are known. 
TABLE 1: Parameters of the example system.

\begin{tabular}{cccccccccccc}
\hline$\varepsilon$ & $\omega_{e}(\mathrm{rad} / \mathrm{s})$ & $a / R$ & $i_{\mathrm{wpi}}$ & $N I_{s}(\mathrm{~A})$ & $r(\mathrm{~mm})$ & $I(\mathrm{~A})$ & $C$ & $D$ & $R(\mathrm{~mm})$ & $z_{1}$ & $L(\mathrm{H})$ \\
\hline 0.25 & 1000 & 2 & 8 & 100 & 25 & 180 & 8707.2 & -0.00066 & 100 & 8 & $1 \times 10^{-3}$ \\
\hline
\end{tabular}

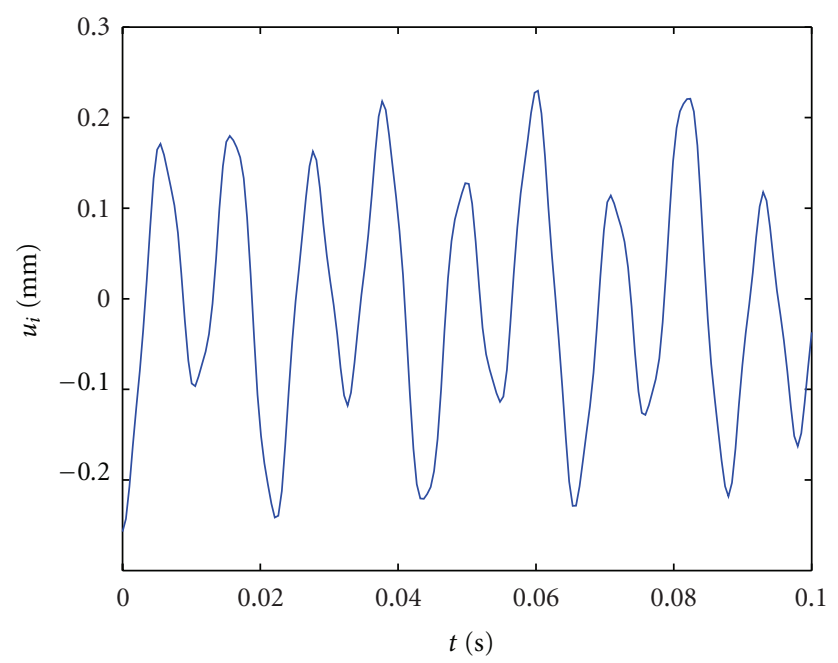

(a) $u_{i}$

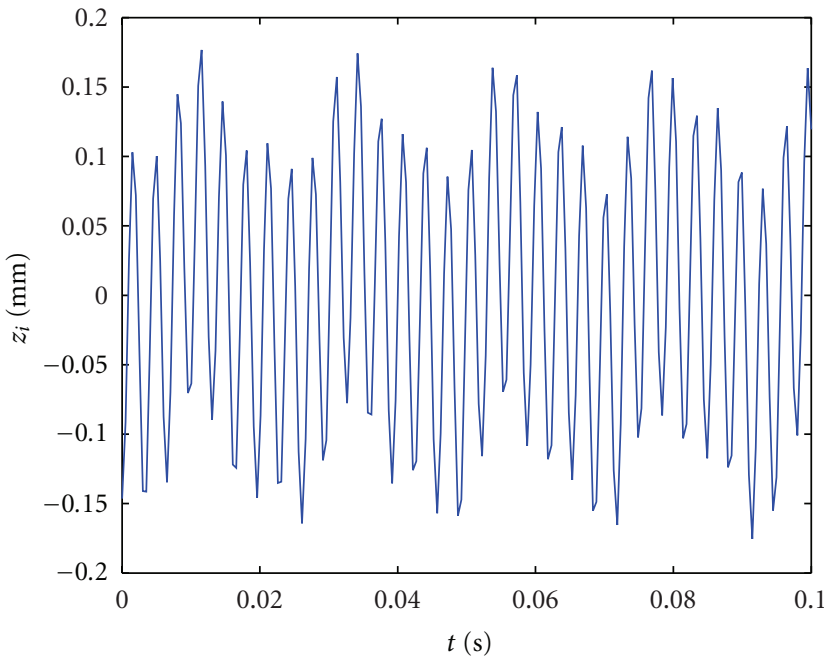

(b) $z_{i}$

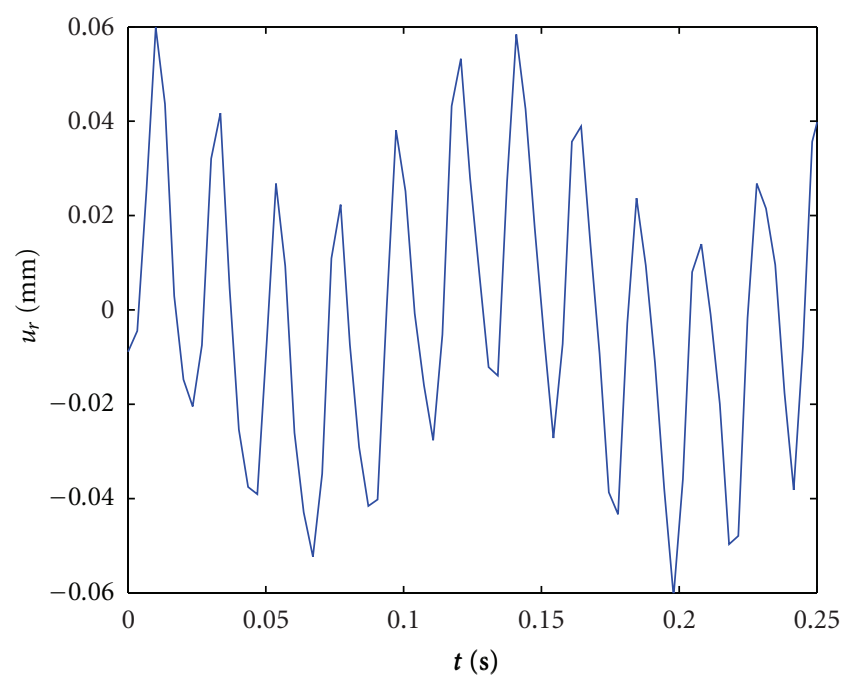

(c) $u_{r}$

FIGURE 4: Nonlinear forced vibration to coupled excitations far from natural frequencies.

(1) Under the coupled excitations, the nonlinear forced responses of the drive system change periodically and unsteadily. The time period of the nonlinear forced responses depends on the frequencies of the electric excitation the mesh parameter excitation, and the nonlinear natural frequencies of the drive system.

(2) The vibrating amplitudes of the nonlinear forced responses of the drive system to the coupled excitations are larger than those of the nonlinear forced responses to the single excitation. The vibrating amplitudes of the nonlinear forced responses of the planet are larger than that of the rotor. It is because the exciting frequencies are near to the vibrating frequency of the planet modes.

(3) The vibrating amplitudes of the tangent vibration for the planet are larger than that of the axial vibration for the planet, and the frequency of the tangent vibration for the planet is smaller than that of the axial vibration for the planet.

(4) The unstable periodic vibrations are harmful for the drive operation. In order to increase the dynamics performance of the drive system, the electric excitations should be avoided or the frequencies of the 


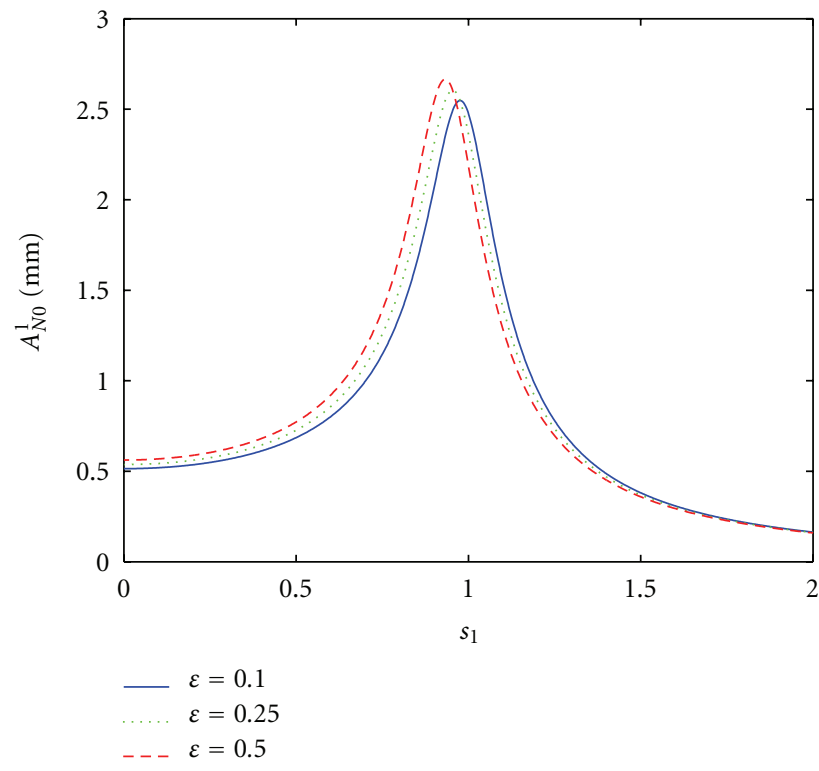

(a) mode 1

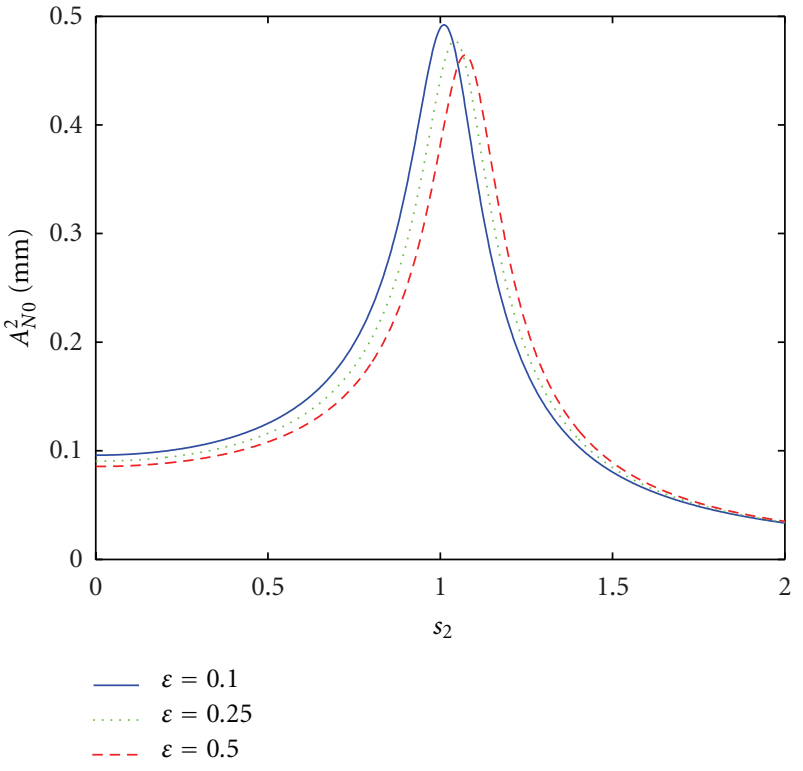

(b) mode 2

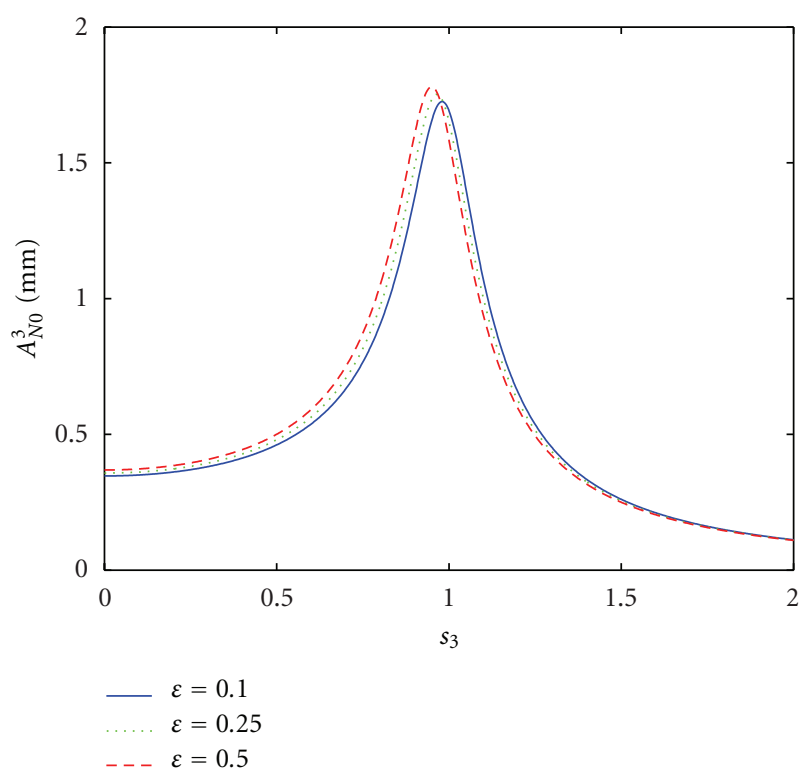

(c) mode 3

FIGURE 5: Nonlinear forced vibration to coupled excitations near natural frequencies.

electric excitations should not be taken as integral multiple of the mesh parameter exciting frequency.

When exciting frequency is near natural frequency, changes of the nonlinear vibrating magnitudes along with exciting frequencies and the drive parameters are given in Figure 5. From Figure 5, the following are known.

(1) As exciting frequency increase, the vibrating magnitudes of all the modes increases, and at point $s_{i}=$ $\omega_{e i} / \omega_{i} \approx 1$, the vibrating magnitudes get to the maximum, and then they decrease with increasing exciting frequency. For different modes, the previously mentioned curves bend toward the direction of the exciting frequency increase. The results are typical nonlinear character of the drive system.

(2) For the first and third modes, as nonlinear parameter $\varepsilon$ increases, their vibrating magnitudes increase. For the second mode, as nonlinear parameter $\varepsilon$ increases, its vibrating magnitude decreases. The vibrating magnitudes are large for the first and third modes and small for the second mode. The vibrating magnitude for the first mode is larger than that for the third mode. 
(3) For a given exciting frequency, the vibrating amplitudes of the nonlinear forced responses of the drive system to the coupled excitations for modes 1 and 3 are larger than those of the nonlinear forced responses to the single excitation; for mode 2 , the vibrating amplitude of the nonlinear forced responses of the drive system to the coupled excitations is smaller than that of the nonlinear forced responses to the single excitation.

(4) Under the coupled excitations, the nonlinearity has obvious effects on the relationship between the frequency and the amplitudes; it should be considered.

\section{Conclusions}

In this paper, the electric excitation and the parameter excitation from mesh stiffness fluctuation are analyzed. The forced response equations of the drive system to the coupled excitations are presented. For the exciting frequencies far from and near to natural frequencies, the forced responses of the drive system to the coupled excitations are investigated. Results show the following.

(1) Under the coupled excitations, the nonlinear forced responses of the drive system change periodically and unsteadily.

(2) The time period of the nonlinear forced responses depends on the frequencies of the electric excitation the mesh parameter excitation, and the nonlinear natural frequencies of the drive system.

(3) In order to improve the dynamics performance of the drive system, the frequencies of the electric excitations should not be taken as integral multiple of the mesh parameter exciting frequency.

\section{Acknowledgment}

This project is supported by the National Natural Science Foundation of China (no. 51075350).

\section{References}

[1] M. R. Kuehnle, “Toroidgetriebe," Urkunde uber die Erteilung des deutschen Patents, 1301682, 1966.

[2] M. R. Kuehnle, H. Peeken, C. Troeder, and S. Cerniak, "The Toroidal drive," Mechanical Engineering, vol. 103, no. 2, pp. 32-39, 1981.

[3] M. R. Kuehnle, "Toroidal transmission and method and apparatus for making and assembling same," United States Patent, 5863273, 1999.

[4] Z. Delin and L. Huamin, "Side surface harmonic stepper motor," Journal of Mechanical Engineering, vol. 29, no. 5, pp. 96-98, 1993 (Japanese).

[5] O. Barth, "Harmonic piezodrive-miniaturized servo motor," Mechatronics, vol. 10, no. 4, pp. 545-554, 2000.

[6] L. Xu, Z. Huang, and Y. Yang, "Contact stress for toroidal drive," Journal of Mechanical Design, Transactions of the ASME, vol. 125, no. 1, pp. 165-168, 2003.
[7] L. Xu and J. Huang, "Torques for electromechanical integrating toroidal drive," Proceedings of the Institution of Mechanical Engineers, Part C, vol. 219, no. 8, Article ID C15704, pp. 801811, 2005.

[8] A. Shaltout and M. Al-Omoush, "Reclosing torques of large induction motors with stator trapped flux," IEEE Transactions on Energy Conversion, vol. 11, no. 1, pp. 84-90, 1996.

[9] H. Y. Kanaan, K. Al-Haddad, and G. Roy, "Analysis of the electromechanical vibrations in induction motor drives due to the imperfections of the mechanical transmission system," Mathematics and Computers in Simulation, vol. 63, no. 3-5, pp. 421-433, 2003.

[10] L. Xu and X. Hao, "Nonlinear forced vibration for electromechanical integrated toroidal drive," International Journal of Applied Electromagnetics and Mechanics, vol. 28, no. 3, pp. 351-369, 2008. 

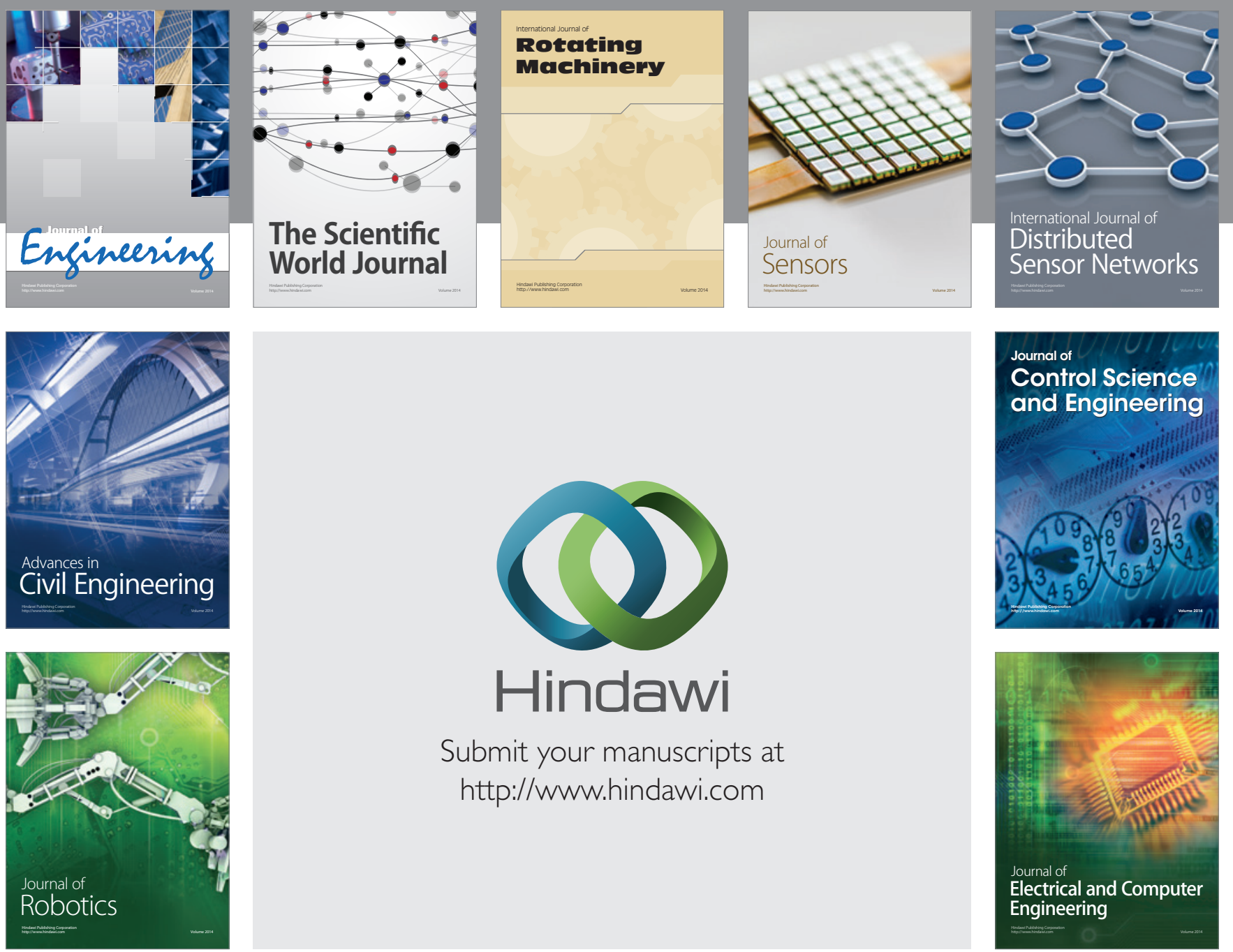

Submit your manuscripts at

http://www.hindawi.com
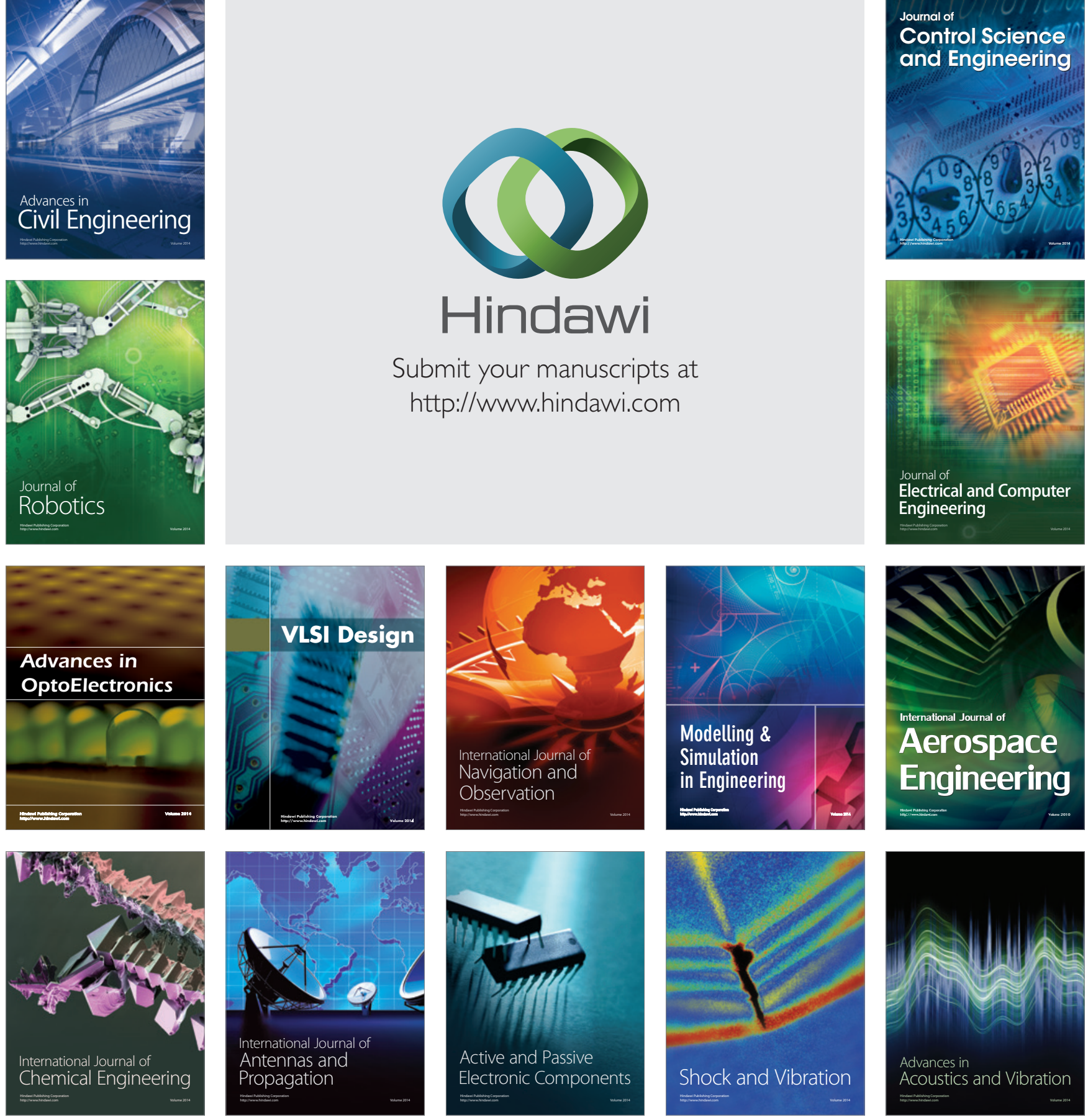